\title{
Intraluminal rectal cancer metastasis to the small bowel: An extremely rare case report
}

\author{
PARASKEVAS STAMOPOULOS ${ }^{1}$, NIKOLAOS MACHAIRAS ${ }^{1}$, STYLIANOS KYKALOS ${ }^{1}$, \\ AFRODITE NONNI ${ }^{2}$, GREGORY KOURAKLIS ${ }^{1}$ and GEORGIOS C. SOTIROPOULOS ${ }^{1}$ \\ ${ }^{1}$ Second Department of Propaedeutic Surgery and ${ }^{2}$ First Department of Pathology, Laiko General Hospital, \\ Athens Medical School, National and Kapodistrian University, 11527 Athens, Greece
}

Received April 11, 2017; Accepted August 4, 2017

DOI: $10.3892 / \mathrm{mco} .2017 .1374$

\begin{abstract}
Prolonged survival in patients suffering from colorectal cancer (CRC) may lead to the emergence of rare metastatic sites that are not well-documented in the literature. We herein describe a very rare case of an intraluminal small intestinal metastasis in a patient with previously resected CRC. A 71-year-old Caucasian male patient with a history of rectosigmoid junction cancer was initially treated with anterior resection. The tumor was classified as pT3pN0 (0/26) M0, stage II. Eighteen months after the primary surgery, local recurrence was detected in the presacral region, and the patient received combined image-guided radiotherapy and chemotherapy. Two months later, due to residual disease at the level of the anastomosis, the patient underwent additional low anterior resection with a diverting stoma. During extensive adhesiolysis, a small palpable intraluminal mass was identified in the jejunum, and segmental small bowel resection was performed. Pathological examination of the resected specimen confirmed that the lesion was a metastasis from the CRC primary. The precise mechanism and clinical significance of $\mathrm{CRC}$ metastasis to the small bowel remain unclear. There is limited clinical experience with this condition, as $<20$ cases have been reported in the literature to date. The main symptoms leading to evaluation, diagnosis and surgical resection are bowel obstruction and bleeding. The present case highlights the possibility of uncomplicated presence of metastatic CRC in the small bowel. Therefore, dilligent inspection of the peritoneal cavity, including the entire length of the gastrointestinal canal, is of paramount importance, particularly in cases of recurrent CRC.
\end{abstract}

Correspondence to: Professor Georgios C. Sotiropoulos, Second Department of Propaedeutic Surgery, Laiko General Hospital, Athens Medical School, National and Kapodistrian University, 17 Agiou Thoma Street, 11527 Athens, Greece

E-mail: georgios.sotiropoulos@uni-due.de

Key words: rectal cancer, colon cancer, small bowel, metastasis, recurrence

\section{Introduction}

Colorectal cancer (CRC) constitutes a considerable cause of morbidity and mortality worldwide, representing the 3rd leading cause of cancer-related mortality in the USA (1). Approximately 135,000 new cases are diagnosed in USA annually, whereas $\sim 50,000$ CRC-related patient deaths are expected per year (1). Over half of the patients with CRC will develop metastases during their lifespan. The most common sites of CRC metastases include the liver, lung and peritoneum; however, various other metastatic sites, such as the bones, spleen, brain and distant lymph nodes, have also been described.

Several improvements and strategic adjustments in the management of patients with metastatic CRC have subsequently led to remarkably improved outcomes (2). Changes in the clinical presentation of the patients, ameliorated preoperative assessment, tailored surgical resection, closer postoperative follow-up and advances in chemotherapeutic regimens represent some of these improvements, which have allowed for expansion of life expectancy in these patients (2). As a consequence, prolonged survival divulges rare metastatic sites that are not well-documented in the literature; the assessment and relevant results from the surgical management of these rare metastatic sites remain ill-reported. We herein describe a very rare case of small intestinal intraluminal metastasis in a patient with a history of previously resected rectosigmoid cancer.

\section{Case report}

A 71-year-old Caucasian male with a history of cancer of the rectosigmoid junction, diagnosed in May 2014, was initially treated with anterior resection. Pathological examination of the surgical specimen revealed moderately differentiated adenocarcinoma, extending through the muscularis propria into the perirectal soft tissue. According to the 7th edition of the Union for International Cancer Control tumor-node-metastasis staging system (inen.sld.pe/portal/documentos/pdf/educacion/13072015_TNM\%20Classification.pdf), the tumor was classified as pT3pN0 (0/26) M0, stage II. Microsatellite instability testing was negative. Following oncological consultation, the patient declined adjuvant chemotherapy. At the 6-month 

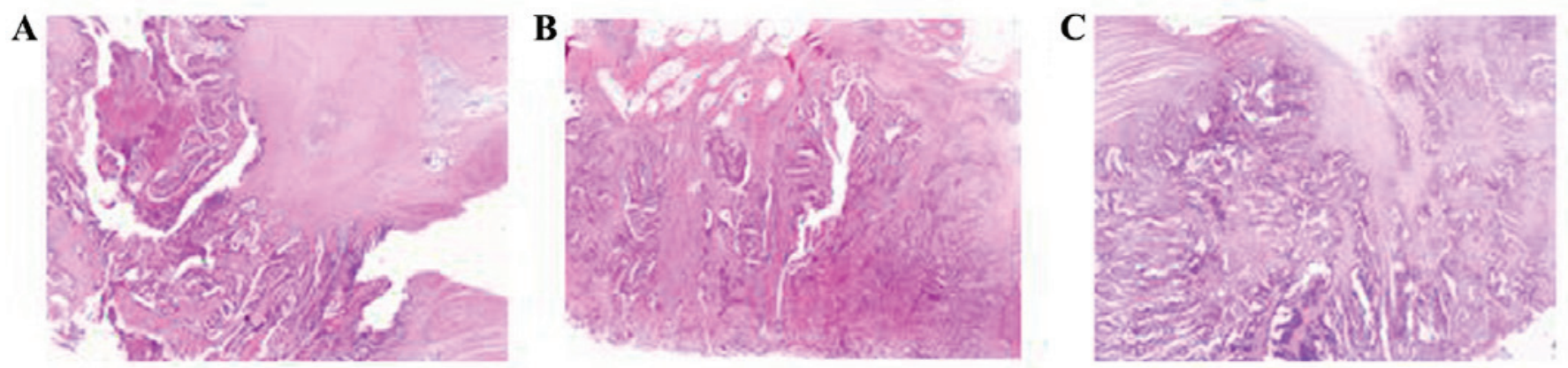

Figure 1. Hematoxylin and eosin staining of paraffin-embedded sections from (A) the primary lesion, (B) the local recurrence, and (C) the small bowel metastasis.

postoperative follow-up, a magnetic resonance imaging (MRI) scan of the abdomen and a computed tomography (CT) scan of the chest were negative for local recurrence or distant metastasis, and the patient underwent laparotomy for repair of an incisional hernia. The intraoperative findings were negative for gross intraperitoneal disease, apart from a small lesion in the presacral space, which proved to be a lymph node negative for cancer infiltration following pathological examination. Eighteen months after the primary surgery, a suspicious lesion was identified in the presacral region on follow-up MRI. Positron emission tomography (PET) confirmed local recurrence. The patient underwent colonoscopy and endoscopic biopsy of the lesion, which was positive for adenocarcinoma, and he received adjuvant chemotherapy (FOLFIRI + bevacizumab) followed by tomotherapy-based image-guided radiotherapy. The patient received a total dose of $54 \mathrm{~Gy}$ to the anastomotic region and $48.6 \mathrm{~Gy}$ to the region of enlarged (but not infiltrated) presacral lymph nodes, discovered during a second laparotomy for hernia repair. A post-interventional MRI revealed complete tumor response according to the Response Evaluation Criteria in Solid Tumors, version 1.1 (iconplc.com/icon-files/docs/IMI\%20 Brochures/2010/Recist-Book-1-1-handout.pdf). The postoperative cross-sectional imaging was negative for distant metastasis. Two months after radiotherapy and 28 months after the initial surgery, a colonoscopy was performed, which revealed a suspicious ulcer at the level of the anastomosis. The obtained biopsies revealed residual disease and the patient was scheduled to receive complementary low anterior resection with total mesorectal excision and a diverting stoma. No gross metastatic disease was detected intraoperatively. During extensive adhesiolysis, a small palpable intraluminal mass was identified in the jejunum, and segmental small bowel resection with end-to-end anastomosis was performed, with low anterior resection and loop ileostomy. Pathological examination revealed local recurrence of the primary rectal adenocarcinoma, without lymph node involvement. The small bowel lesion proved to be a moderately differentiated adenocarcinoma, with morphological characteristics similar to those of the primary and recurrent rectal lesions, invading the entire bowel wall, with metastasis to two regional lymph nodes. Both lesions shared the same immunohistochemical reactivity pattern as colonic adenocarcinoma, with diffuse and strong positive staining for anti-cytokeratin (CK) 20, focal positive staining for anti-CK7, and partial $\alpha$-methylacyl coenzyme A racemase expression. The abovementioned similarities strongly suggested the metastatic nature of the small intestinal lesion. The staining results of the paraffin-embedded thin tumor sections from the primary lesion, the local recurrence and the small bowel metastasis are presented in Figs. 1 and 2. The postoperative course was uneventful. The patient remained disease-free at the time of the last follow-up, which was performed 6 months after admission. The patient provided written informed consent regarding the publication of the case details and associated images. The presentation of this case was approved by the Ethics Committee of the Laiko University Hospital (Athens, Greece) and conformed to the ethical guidelines of the 1975 Declaration of Helsinki.

\section{Discussion}

The most common sites of metastatic CRC include the liver, lung, peritoneum, retroperitoneal sites, and locoregional or peripheral lymph nodes (3). Small bowel tumors represent only $2 \%$ of all gastrointestinal neoplasms (4), and mainly comprise primary tumors, such as adenocarcinomas, gastrointestinal stromal tumors, carcinoids and lymphomas. Metastasis to the small bowel is rare, and mainly originates from melanoma, and breast or lung cancer. The exact mechanism of metastasis to the small bowel has not been fully elucidated, but possible metastatic routes include hematogenous spread, peritoneal spread per continuitatem, or translocation of tumor cells within the intenstinal tract and implantation into the mucosa of the small bowel. The major route of metastasis of CRC to the small bowel is disseminated metastasis, referred to as peritonitis carcinomatosa (5).

There is limited experience reported in the literature describing this medical condition complicating the course of CRC (Table I). Kojima et al reported the case of a small bowel metastasis from cancer of the descending colon, which presented with melena 2 years after the initial colon resection (6). The authors additionally reported 6 more similar cases registered in Japan. Synchronous metastasis was observed in 2 cases, while the mean time of metachronous metastasis was 7 and 36 months after surgery. The main symptoms leading to evaluation, diagnosis and surgical resection were bowel obstruction and bleeding (6). Another study reported a series of 3 cases with metachronous metastatic small bowel cancer originating from CRC. All the patients were referred for obscure gastrointestinal bleeding and underwent capsule endoscopy (CE) with subsequent double-balloon enteroscopy (7). Spada et al analyzed the database of all patients 
Table I. Reported cases of metastatic small bowel tumor from colorectal cancer.

\begin{tabular}{|c|c|c|c|c|c|c|c|}
\hline First author, year & Cases & Age (sex) & Symptoms (n) & $\begin{array}{c}\text { Primary } \\
\text { location (n) }\end{array}$ & $\begin{array}{c}\text { Metastatic } \\
\text { location }\end{array}$ & $\begin{array}{c}\text { Primary resection } \\
\text {-to-metastasis } \\
\text { interval }\end{array}$ & (Refs.) \\
\hline Kojima, 2010 & 7 & NA & $\begin{array}{l}\text { Obstruction (4), } \\
\text { melena (1), } \\
\text { fatigue }(1), \\
\text { none (1) }\end{array}$ & $\begin{array}{l}\text { Right colon }(1), \\
\text { transverse colon }(1), \\
\text { left colon }(4), \\
\text { rectum }(1)\end{array}$ & $\begin{array}{c}\text { Jejunum (5) } \\
\text { Ileum (2) }\end{array}$ & $\begin{array}{c}\text { Synchronous (2) } \\
\text { Mean, } 43 \\
\text { months (5) }\end{array}$ & (6) \\
\hline Rondomonti, 2008 & 2 & $\begin{array}{c}56(\mathrm{M}) \\
\text { NA }\end{array}$ & $\begin{array}{c}\text { Bleeding } \\
\text { NA }\end{array}$ & $\begin{array}{l}\text { NA } \\
\text { NA }\end{array}$ & $\begin{array}{l}\text { Ileum } \\
\text { NA }\end{array}$ & $\begin{array}{l}1 \text { month } \\
\text { NA }\end{array}$ & (10) \\
\hline Thoma, 2011 & 3 & $\begin{array}{l}73(\mathrm{~F}) \\
56(\mathrm{M}) \\
73(\mathrm{M})\end{array}$ & $\begin{array}{l}\text { Anemia } \\
\text { Bleeding } \\
\text { Bleeding }\end{array}$ & $\begin{array}{l}\text { Right colon } \\
\text { Rectum } \\
\text { Left colon }\end{array}$ & $\begin{array}{l}\text { Ileum } \\
\text { Ileum } \\
\text { Jejunum }\end{array}$ & $\begin{array}{c}7 \text { years } \\
15 \text { months } \\
3 \text { years }\end{array}$ & (7) \\
\hline Bailey, 2006 & 1 & NA & Bleeding & NA & NA & NA & (9) \\
\hline Spada, 2008 & 3 & $\begin{array}{l}67(\mathrm{M}) \\
45(\mathrm{M}) \\
72(\mathrm{~F})\end{array}$ & $\begin{array}{l}\text { Bleeding } \\
\text { Bleeding } \\
\text { Bleeding }\end{array}$ & $\begin{array}{l}\text { NA } \\
\text { NA } \\
\text { NA }\end{array}$ & $\begin{array}{l}\text { Ileum } \\
\text { Ileum } \\
\text { Jejunum }\end{array}$ & $\begin{array}{l}\text { NA } \\
\text { NA } \\
\text { NA }\end{array}$ & (8) \\
\hline Meshikes, 2016 & 1 & $67(\mathrm{M})$ & Obstruction & Left colon & Jejunum & 3 years & (11) \\
\hline Huerta, 2009 & 1 & NA & Obstruction & NA & NA & $>3$ years & (13) \\
\hline Iwamuro, 2015 & 1 & $57(\mathrm{M})$ & None & Sigmoid colon & Duodenum & NA & $(12)$ \\
\hline
\end{tabular}

M, male; F, female; NA, not available.
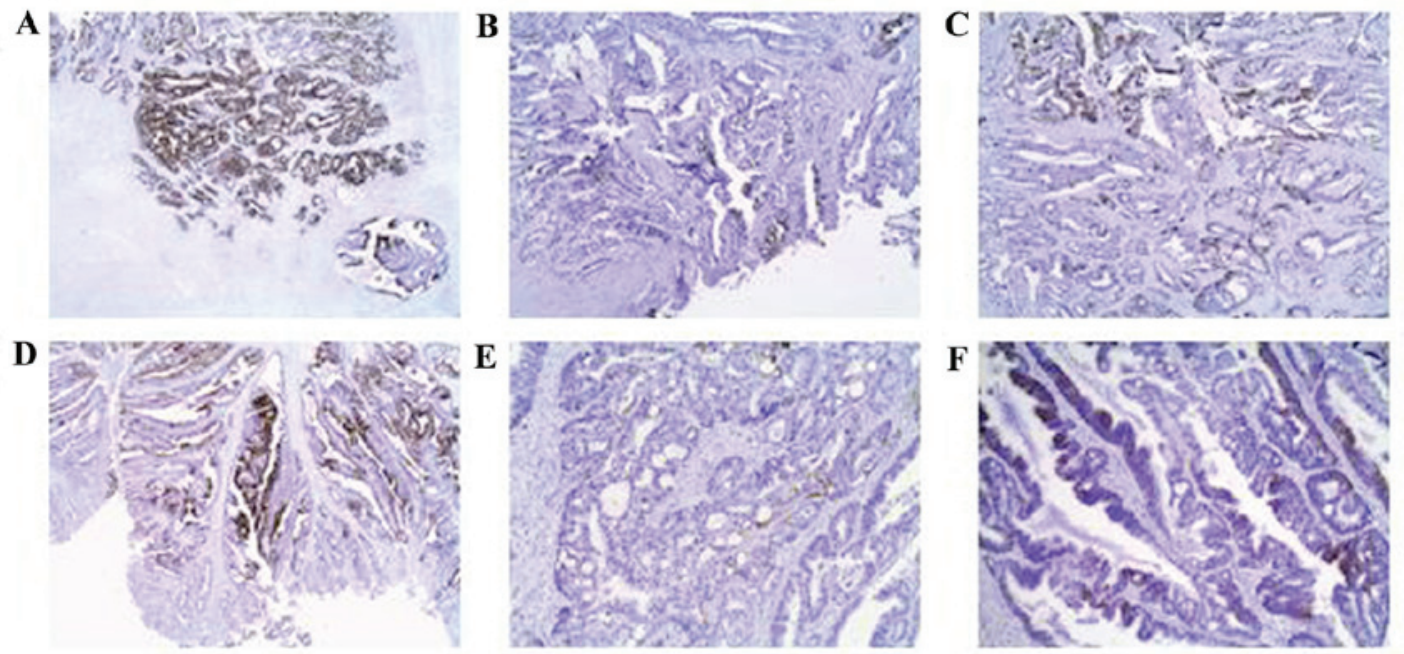

Figure 2. (A-F) Immunohistochemical profile of recurrent large bowel tumor and its small bowel metastasis for the CK7, CK20 and AMACR markers (A and D) CK20 was strongly expressed, (B and E) CK7 was focally expressed, while (C and F) AMACR was partially expressed in recurrent large bowel tumor and its small bowel metastasis, respectively. CK, cytokeratin; AMACR, $\alpha$-methylacyl coenzyme A racemase.

referred to their center for CE. A malignant small bowel tumor was identified in 13 of the 380 cases. In 3 of these cases, the histological examination of the resected specimens confirmed the presence of metachronous small bowel metastasis of a known colonic adenocarcinoma. All 3 patients had been referred for obscure gastrointestinal bleeding, while the metastatic site was located at the level of the ileum in 2 patients and at the level of the jejunum in the third patient (8). Two more multicentric studies from Europe and Australia analyzed findings from $\mathrm{CE}$ in patients with small bowel symptoms and reported 3 additional cases with CRC metastasis to the small bowel $(9,10)$. Furthermore, there are 3 more referrals of isolated cases with small bowel metastases from CRC, one of which was located in the duodenum (11-13).

The clinical presentation of small bowel tumors is mostly atypical; these lesions grow slowly, remain asymptomatic over a long period of time and cause signs later during the clinical course. The non-specific manifestations include vague abdominal pain, maldigestion, bleeding, anemia or acute obstruction. Extraintestinal symptoms in the setting of para- 
neoplastic syndromes may also be present (14). The majority of reported metachronous small bowel metastases have been in fact diagnosed upon development of similar complications (i.e., lower gastrointestinal bleeding or bowel obstruction). Contrary to those cases, the intraluminal CRC metastasis to the small bowel in the present case was incidentally identified during re-operation for local recurrence of the primary tumor.

The diagnosis of small bowel lesions is primarily based on radiological and endoscopic findings. Small bowel tumors may be detected on standard abdominal CT. In case of unclear or highly suspicious findings, CT enterography, MRI enterography or enteroclysis are recommended. The choice between MRI and CT relies upon the clinician's discretion, although MRI is favored, given the absence of radiation (15). PET-CT is not the examination of first choice, although it may be helpful in case of equivocal findings on CT/MRI, or in cases with suspected metastatic disease. The assessment of the entire length of the small bowel may be difficult, as gastroduodenoscopy and colonoscopy facilitate the examination of only short portions of the proximal and distal small intestine, respectively. The evolution of endoscopic techniques (video CE and single or double balloon-assisted enteroscopy) have allowed for direct visualization of the entire small intestine (16). In most of the abovementioned reported cases of small bowel metastases, the diagnosis was established based on the findings of $\mathrm{CE}$. Although this procedure is widely available, it presents several critical limitations, such as inability to obtain biopsies, identify intramural or extraluminal lesions and perform therapeutic interventions, as well as the risk of capsule retention during the examination (9). Of note, the miss rate of $\mathrm{CE}$ in detecting tumor lesions may be as high as $18.9 \%$ (17).

CRC metastasis to the small bowel represents a rare and usually late complication of the disease. Such a condition represents a negative prognostic factor regarding disease progression and patient survival. The survival rates following resection of CRC metastasis to the small bowel are encouraging. Only 2 patients succumbed to disease progression at 1 and 26 months postoperatively $(6,9,11)$. Furthermore, a case of disease-free survival of $>11$ years has also been reported (6). Whether simple $\mathrm{R} 0$ resection via limited enterectomy is oncologically equivalent to radical resection (with concomitant removal of the segmental mesentery) remains to be elucidated.

The present case highlights the possibility of uncomplicated presence of metastatic CRC to the small bowel, which may be underestimated in surgical patients with a history of CRC. Thorough inspection of the entire peritoneal cavity, including the whole length of the gastrointestinal canal, is of paramount importance in cases of CRC recurrence. Moreover, thorough pathological and immunohistochemical examination is mandatory for differentiating metastatic from primary synchronous small bowel carcinomas. Future studies are required to address certain critical issues with regards to this clinical entity, such as whether simple R0 small bowel resection is oncologically adequate for these patients. Furthermore, it remains to be determined whether CRC metastases to this rare site greatly affect long-term survival in such patients. To the best of our knowledge, this is the first report of an incidentally identified intraluminal $\mathrm{CRC}$ metastasis to the small bowel during re-operation for recurrence.
Surgeons should be aware of this uncommon intraoperative finding in patients previously treated for CRC. An aggressive surgical approach to such lesions, when identified, will provide valuable information on the true incidence and natural course of metastatic CRC to the small bowel.

\section{References}

1. Siegel RL, Miller KD and Jemal A: Cancer statistics, 2016. CA Cancer J Clin 66: 7-30, 2016.

2. Van Cutsem E, Cervantes A, Adam R, Sobrero A, Van Krieken JH, Aderka D, Aranda Aguilar E, Bardelli A, Benson A, Bodoky G, et al: ESMO consencus guidelines for the management of patients with metastatic colorectal cancer. Ann Oncol 27: 1386-1422, 2016.

3. Figueredo A, Rumble RB, Maroun J, Earle CC, Cummings B, McLeod R, Zuraw L and Zwaal C; Gastrointestinal Cancer Disease Site Group of Cancer Care Ontario's Program in Evidence-based Care: Follow-up of patients with curatively resected colorectal cancer: A practice guideline. BMC Cancer 3: 26, 2003.

4. Reynolds I, Healy P and Mcnamara DA: Malignant tumours of the small intestine. Surgeon 12: 263-270, 2014.

5. DeCastro CA, Dockerty MB and Mayo CW: Metastatic tumors of the small intestines. Surg Gynecol Obstet 105: 159-165, 1957.

6. Kojima Y, Matsumoto F, Mikami Y, Namekata K and Takei M: Metastatic small bowel tumor from descending colon cancer with extensive hematogenous or lymphogenous spread: Survey of the japanese literature. Case Rep Gastroenterol 4: 340-345, 2010.

7. Thoma MN, Saiyed SM and Charles RJ: Metastatic colorectal cancer to the small bowel-an uncommon cause of obscure GI bleeding: A three-case experience with review of the literature. J Gastrointest Cancer 42: 119-122, 2011.

8. Spada C, Riccioni ME, Familiari P, Marchese M, Bizzotto A and Costamagna G: Video capsule endoscopy in small-bowel tumours: A single centre experience. Scand J Gastroenterol 43: 497-505, 2008

9. Bailey AA, Debinski HS, Appleyard MN, Remedios ML, Hooper JE, Walsh AJ and Selby WS: Diagnosis and outcome of small bowel tumors found by capsule endoscopy: A three-center Australian experience. Am J Gastroenterol 101: 2237-2243, 2006.

10. Rondonotti E, Pennazio M, Toth E, Menchen P, Riccioni ME, De Palma GD, Scotto F, De Looze D, Pachofsky T, Tacheci I, et al: Small-bowel neoplasms in patients undergoing video capsule endoscopy: A multicenter European study. Endoscopy 40: 488-495, 2008.

11. Meshikhes AW and Joudeh AA: Late metastatic colon cancer masquerading as primary jejunal carcinoma. Ann R Coll Surg Engl 98: e49-e51, 2016.

12. Iwamuro $M$, Uetsuka $H$, Makihata $K$ and Yamamoto $K$ : Metastatic tumors in the duodenum: A report of two cases. J Cancer Res Ther 11: 648, 2015.

13. Huerta S and Li HC: Small bowel obstruction due to metastatic colon cancer. Anticancer Drugs 20 Spec No 2: S15-S18, 2009.

14. Talamonti MS, Goetz LH, Rao S and Joehl RJ: Primary cancers of the small bowel: Analysis of prognostic factors and results of surgical management. Arch Surg 137: 564-571, 2002.

15. Murphy KP, McLaughlin PD, O'Connor OJ and Maher MM: Imaging the small bowel. Curr Opin Gastroenterol 30: 134-140, 2014.

16. Ma JJ, Wang Y, Xu XM, Su JW, Jiang WY, Jiang JX, Lin L, Zhang DQ, Ding J, Chen L, et al: Capsule endoscopy and single-balloon enteroscopy in small bowel diseases: Competing or complementary?. World J Gastroenterol 22: 10625-10630, 2016.

17. Lewis BS, Eisen GM and Friedman S: A Pooled analysis to evaluate results of capsule endoscopy trials. Endoscopy 37: 960-965, 2005. 